\title{
O ensino de implantodontia nas graduações brasileiras de odontologia: um estudo transversal
}

\author{
The teaching of implant dentistry in Brazilian dentistry graduations: a cross-sectional study
}

La enseñanza de la implantología en las graduaciones de odontología brasileña: un estudio transversal

Luan Viana Faria ${ }^{1 *}$, Gisele Aparecida de Oliveira ${ }^{1}$, Maria Eduarda Povoleri Grázzia1, Yuri de Lima Medeiros ${ }^{1}$, Danielle Fernandes Lopes ${ }^{1}$, Isabel Cristina Gonçalves Leite ${ }^{2}$.

\section{RESUMO}

Objetivo: Fornecer uma visão geral da atual oferta da disciplina de implantodontia nos cursos de Odontologia das Instituições de Ensino Superior (IES) brasileiras, incluindo as características curriculares da disciplina. Métodos: Foram analisadas as grades curriculares disponibilizadas em ferramentas online, como os sítios web oficial ou os e-mails de coordenadores de cursos de todas as IES de odontologia dos estados sul e sudeste do Brasil, cadastradas no portal e-MEC do Ministério da Educação. Resultados: 36 (16,51\%) eram escolas públicas e $182(83,48 \%)$ privadas, sendo que IES públicas oferecem mais a disciplina que IES privadas $(86,1 \%$ contra $54,9 \%, p<0,001)$. Quando administrado, o conteúdo de implantodontia se dá de forma teórica em mais de $60 \%$ dos casos $(p=0,723)$, independente da natureza do curso. Tanto nas públicas quanto nas privadas, a disciplina é mais frequentemente ofertada como obrigatória, $67,7 \%$ e $92,3 \%$ respectivamente, embora com frequências estatisticamente diferentes $(<0,001)$. A carga horária média é de $60,97 \mathrm{~h}$, nas públicas é de 53,29h ( $D P=23,943)$ e nas privadas de 63,99h ( $D P=34,627)$. Conclusão: Grande parte das IES da região sul e sudeste do Brasil, especialmente as instituições particulares, ainda não ofertam a disciplina de implantodontia. Quando ofertada, a disciplina tende a ser obrigatória e teórica.

Palavras-chave: Implantes dentários, Educação em odontologia, Ensino, Currículo, Odontologia.

\section{ABSTRACT}

Objective: To provide an overview of the current offer of implant dentistry in the Dentistry courses of Brazilian Higher Education Institutions (HEls), including the curricular characteristics of the discipline. Methods: Curriculum frameworks made available through online tools, such as the official websites or e-mails of course coordinators from all HEls in the southern and southeastern states of Brazil, registered in the e-MEC portal of the Ministry of Education, were analyzed. Results: 36 (16.51\%) were public schools and $182(83.48 \%)$ private, with public HEls offering more discipline than private HEls $(86.1 \%$ versus $54.9 \%, p<0.001)$. When administered, the implant dentistry content is theoretical in more than $60 \%$ of cases $(p=0.723)$, regardless of the nature of the course. In both public and private, the discipline is most often offered as compulsory, $67.7 \%$ and $92.3 \%$ respectively, although with statistically different frequencies $(<0.001)$. The average workload is $60.97 \mathrm{~h}$, in the public hours 53.29h $(S D=23.943)$ and in the private $63.99 \mathrm{~h}(\mathrm{SD}=34.627)$. Conclusion: Most HEls in southern and southeastern Brazil, especially private institutions, do not offer the discipline of implant dentistry. When offered, discipline tends to be compulsory and theoretical.

Key words: Dental implants, Education dental, Teaching, Curriculum, Dentistry.

1 Universidade Federal de Juiz de Fora (UFJF), Juiz de Fora - MG.

*E-mail: luanvfaria13@hotmail.com 


\section{RESUMEN}

Objetivo: Proporcionar una visión general de la oferta actual de implantología dental en los cursos de Odontología de las Instituciones de Educación Superior (IES) de Brasil, incluidas las características curriculares de la disciplina. Métodos: se analizaron los marcos curriculares disponibles a través de herramientas en línea, como los sitios web oficiales o los correos electrónicos de los coordinadores de cursos de todas las IES dentales en los estados del sur y sureste de Brasil, registrados en el portal e-MEC del Ministerio de Educación. Resultados: 36 (16.51\%) eran escuelas públicas y 182 (83.48\%) eran privadas, con IES públicas que ofrecían más disciplina que las IES privadas $(86.1 \%$ versus $54.9 \%, p<0.001)$. Cuando se administra, el contenido de la odontología del implante es teórico en más del $60 \%$ de los casos $(p=0,723)$, independientemente de la naturaleza del curso. Tanto en público como en privado, la disciplina se ofrece con mayor frecuencia como obligatoria, $67.7 \%$ y $92.3 \%$ respectivamente, aunque con frecuencias estadísticamente diferentes $(<0.001)$. La carga de trabajo promedio es $60.97 \mathrm{~h}$, en las horas públicas $53.29 \mathrm{~h}$ $(S D=23.943)$ y en las privadas $63.99 h(S D=34.627)$. Conclusión: La mayoría de las IES en el sur y sureste de Brasil, especialmente las instituciones privadas, aún no ofrecen la disciplina de la implantología. Cuando se ofrece, la disciplina tiende a ser obligatoria y teórica.

Palabras clave: Implantes dentales, Educación en odontología, Enseñanza, Curriculum, Odontología.

\section{INTRODUÇÃO}

Nas últimas décadas, a implantodontia tem crescido continuamente em importância para a reabilitação dentária. Graças aos avanços tecnológicos e às pesquisas, tanto a indicação quanto o uso de implantes bucais aumentaram (KOOLE S e DE BRUYN H, 2014; VANDEWEGHE S, et al., 2014). Como alternativas às opções convencionais de tratamento restaurador, a terapia com implantes tornou-se uma parte importante do tratamento para restaurar a função e a estética em pacientes total ou parcialmente desdentados (SANZ M e SAPHIRA L, 2009). Passando de terapia experimental, que ocupava um determinado nicho e praticada por um seleto grupo de profissionais em universidades ou centros especializados, atualmente se constitui como uma terapia mais acessível e de alcance global que expande exponencialmente (VANDEWEGHE S, et al., 2014; DRAGAN IF, et al., 2019).

Para atender aos padrões ideais de uma dentição funcional, mais pacientes necessitam de odontologia reconstrutiva, frente a situações de edentulismo total e parcial. O constante aprimoramento das técnicas protéticas, dos materiais dos implantes e protocolos cirúrgicos tornaram a terapia com implantes uma alternativa valiosa e previsível de tratamento da odontologia reconstrutiva. Do ponto de vista biológico, o implante também é a primeira opção de tratamento para a substituição de um dente (KOOLE S e DE BRUYN $\mathrm{H}$, 2014). A terapia com implantes adquiriu grande sucesso clínico na prática odontológica moderna, embalada pela relação custo-benefício em longo prazo quando comparado a outros tratamentos reabilitadores protéticos (PAPASPYRIDAKOS P, et al., 2014), pelas altas taxas de sucesso geralmente observadas (KROEPLIN BS e STRUB JR, 2011), pela melhoria das medidas de qualidade de vida dos pacientes tratados (FILLION M, et al., 2013) e pelas relativas baixas taxas de complicações (BRÄGGER U, et al., 2005).

Baseado no aumento da demanda de pacientes para tratamento com implantes, a necessidade de uma educação padronizada e estruturada em implantodontia foi reconhecida por consenso global entre clínicos, pesquisadores e educadores (KOOLE S e DE BRUYN H, 2014; JAHANGIRI L, 2015). A promoção de workshops de consenso por organizações representativas de instituições odontológicas americanas (PETROPOULOS VC, et al., 2006), europeias (LANG NP e DE BRUYN H, 2009) e australianas (MATTHEOS N e IVANOVSKI S, 2010; MATTHEOS N, et al., 2010) legitimaram a necessidade de uma maior inclusão de terapia com implantes ao nível da graduação odontológica. A maioria das instituições acadêmicas norteamericanas e europeias, nos últimos anos, estabeleceu programas dedicados ao ensino de implantes dentários no nível acadêmico, em um esforço para preparar clínicos gerais para o fornecimento de terapia restauradora de implantes dentários de rotina (CHRISTOPHER A, et al., 2015). No Brasil, um único estudo realizado em 2010 , observou que $43,04 \%$ das faculdades pesquisadas não dispunham da disciplina de

REAS/EJCH | Vol.12(4) | e2672 | DOI: https://doi.org/10.25248/reas.e2672.2020 Página 2 de 10 
Implantodontia (RABELO CC, et al., 2010). Nas Instituições de Ensino Superior (IES) brasileiras onde a disciplina inexiste, os conhecimentos sobre a Implantodontia são ministrados, na maioria das vezes $(53,84 \%)$, na disciplina de Cirurgia Bucomaxilofacial, o que, analisando criticamente, pode contribuir para o ensino superficial e fragmentado do assunto.

Qualquer que seja o nível de habilidades clínicas ensinado aos alunos de graduação, é consenso que futuros cirurgiões-dentistas devem se comunicar com outros membros da equipe em relação ao planejamento do tratamento e à resolução de problemas relacionados ao paciente (PLASSCHAERT AJ, et al., 2005). Por isso, é necessário que a implantodontia esteja incluída nos currículos de graduação em odontologia, uma vez que se torna indispensável para a boa formação e inserção profissional no mercado de trabalho.

De modo geral, é oportuno examinar como as inovações na prática odontológica são introduzidas nos currículos de graduação já sobrecarregados. Baseado nesses aspectos, o objetivo deste estudo é fornecer uma visão geral da atual oferta da disciplina de implantodontia nas graduações das IES brasileiras, incluindo características curriculares como natureza da disciplina e carga horária, bem como discutir a sua importância na grade curricular do curso de graduação.

\section{MÉTODOS}

A técnica metodológica proposta foi baseado segundo estudo de base documental, através de busca da presença da disciplina de implantodontia na matriz ou grade curricular das IES do Sudeste e Sul do Brasil, escolhidas por serem as regiões brasileiras com menor relação habitante/cirurgião-dentista, o que reflete a existência de um mercado com excesso de profissionais (MARTIN ASS, et al., 2018). As IES que ofertam o curso de odontologia foram identificadas no Cadastro Nacional de Cursos e Instituições de Educação Superior - Cadastro e-MEC (BRASIL, 2019), que é regulamentado pela Portaria Normativa no 21, de 21 de dezembro de 2017, que dispõe sobre o sistema eletrônico de fluxo de trabalho e gerenciamento de informações relativas aos processos de regulação, avaliação e supervisão da educação superior no sistema federal de educação, e o Cadastro Nacional de Cursos e Instituições de Educação Superior Cadastro e-MEC (BRASIL, 2017). A plataforma e-MEC pode ser acessada via endereço eletrônico <www.emec.mec.gov.br>. A coleta desses dados foi realizada em março de 2019, na qual foram catalogadas e tabeladas todas as instituições dispostas no sistema.

Como critério de inclusão, considerou-se todos os cursos de Odontologia em funcionamento, que estavam com cursos iniciados e credenciados pelo Ministério da Educação e Cultura (MEC) na região sudeste e região sul do Brasil. Para confirmar a existência da disciplina de Implantodontia, realizou-se a busca em todos os sites institucionais a procura da matriz ou grade curricular mais recente do curso de Odontologia. Na instituição em que não foi possível encontrar a matriz curricular por este meio, os coordenadores foram contatados por e-mail, solicitando a disponibilização da grade curricular. Foi aguardado um prazo de dois meses para que os e-mails fossem respondidos. Foram excluídas as IES que não possuíam um sítio web oficial e que não forneciam meios de comunicação virtual, assim como aquelas que não apresentaram a grade curricular completa disponível, em ambos canais. O período de coleta das matrizes curriculares foi de março a junho de 2019.

Foram objetos de estudo as seguintes informações: (I) categoria administrativa da IES: pública ou privada; (II) inserção e oferta da disciplina: presença ou ausência; (III) natureza do componente curricular: obrigatória ou não-obrigatória; (IV) metodologia de ensino: conteúdo teórico ou teórico-prático e (V) carga horária total $(\mathrm{CH})$. Foram considerados diferentes polos de uma mesma instituição, bem como instituições que ofertavam o curso de Odontologia em mais de uma modalidade, se integral ou noturno, foram tratadas como dados independentes, uma vez que a matriz curricular pode variar entre essas situações, mesmo quando sendo pertencentes a uma mesma instituição. As IES que possuíam mais de uma disciplina reservada para o tema tiveram as cargas horárias somadas, independente se ofertadas anualmente ou semestralmente.

Os softwares estatísticos utilizados na análise das variáveis e confecção dos gráficos foram o GraphPad Prism 8.1.2 (GraphPad Software Inc., La Jolla, CA, EUA) e Statistical Package for Social Sciences® 15.0 
(SPSS Inc., Chicago, IL, EUA), sendo analisados por meio de estatísticas descritivas. O teste estatístico utilizado para comparação de proporções foi o Qui-quadrado de Pearson. Para a variável (V) carga horária $(\mathrm{CH})$, por apresentarem distribuição normal de acordo com o teste Kolmogorov-Smirnov, a opção de comparação eleita foi o Teste-t e teste de Levene para igualdade de variâncias visando a comparação entre cargas horárias, segundo (I) categoria administrativa da IES e (IV) metodologia de ensino. O nível de significância adotado foi de $5 \%$.

\section{RESULTADOS}

Os resultados desta pesquisa refletem a análise da grade curricular de 218 IES localizadas nos estados do sudeste e sul brasileiro (Tabela 1). Das 260 Instituições cadastradas no portal e-MEC, 218 compuseram a amostra final deste estudo por concederem acesso as grades curriculares, sendo 144 instituições localizadas na região sudeste e 74 na região Sul. Dentre elas, 10 IES se localizam no estado do Espirito Santo (ES), 51 em Minas Gerais (MG), 21 no Rio de Janeiro (RJ), 62 em São Paulo (SP), 28 no Paraná (PR), 37 no Rio Grande do Sul (RS) e 21 no estado de Santa Catarina.

Tabela 1 - Total de grades curriculares das IES analisadas por estado.

\begin{tabular}{lcccc}
\hline & $\begin{array}{c}\text { Total de IES } \\
(\mathbf{n = 2 6 0 )}\end{array}$ & $\begin{array}{c}\text { Instituições } \\
\text { Incluídas (n=218) }\end{array}$ & $\begin{array}{c}\text { IES públicas } \\
\text { incluídas }\end{array}$ & $\begin{array}{c}\text { IES privadas } \\
\text { incluídas }\end{array}$ \\
\hline Região Sudeste & $\mathbf{1 7 6}$ & $\mathbf{1 4 4}(\mathbf{8 1 , 8 \% )}$ & $\mathbf{1 9}$ & $\mathbf{1 2 5}$ \\
\hline Espírito Santo (ES) & 12 & $10(83,3 \%)$ & 1 & 9 \\
Minas Gerais (MG) & 61 & $51(83,6 \%)$ & 7 & 44 \\
Rio de Janeiro (RJ) & 28 & $21(75,0 \%)$ & 4 & 17 \\
São Paulo (SP) & 75 & $62(82,6 \%)$ & 7 & 55 \\
\hline Região Sul & $\mathbf{8 4}$ & $\mathbf{7 4}(\mathbf{8 8 , 0 \% )}$ & $\mathbf{1 7}$ & 22 \\
\hline Paraná (PR) & 37 & $28(75,6 \%)$ & 6 & 20 \\
Rio Grande do Sul (RS) & 25 & $25(100 \%)$ & 5 & 15 \\
\hline Santa Catarina (SC) & 22 & $21(95,4 \%)$ & 6 & \\
\hline
\end{tabular}

Fonte: Faria LV, et al., 2019.

Com relação à (I) categoria administrativa das IES, 36 (16,51\%) eram públicas e 182 (83,48\%), privadas. As regiões sul e sudeste apresentaram uma maior tendência a ter mais cursos privados do que públicos, mas não foi estatisticamente significativo (Tabela 2). Ainda observando regionalmente, quanto à oferta, as IES de odontologia apresentam a disciplina em mais de $58 \%$ das grades curriculares, e quando ofertam, a disciplina é predominantemente obrigatória.

Quanto à metodologia aplicada, o conteúdo da disciplina é apenas teórico na maioria das escolas do sudeste, enquanto na região sul observa-se que metade das instituições incluem em seu currículo alguma prática relacionada à disciplina de implantodontia. Do ponto de vista regional, para as variáveis (II) oferta, (III) natureza e (IV) metodologia de ensino, não é observado diferenças entre as regiões. Ao comparar as variáveis (II) oferta, (III) natureza e (IV) metodologia de ensino de acordo com a variável (I) categoria administrativa, 
como demostrado (Tabela 3), observa-se que IES públicas oferecem mais a disciplina $(86,1 \%)$ que IES privadas $(54,9 \%)$. A única variável que não apresentou diferença estatística foi a distribuição quanto ao conteúdo, se teórico ou prático (Tabela 3).

Tabela 2 - Prevalência da disciplina de implantodontia nas regiões sul e sudeste do Brasil, 2019.

\begin{tabular}{|c|c|c|c|c|c|}
\hline Variáveis & $\begin{array}{c}\text { Região sudeste } \\
\text { N }\end{array}$ & $\begin{array}{c}\text { Região sul } \\
\mathbf{N}\end{array}$ & $\begin{array}{c}\text { Região sudeste } \\
\%\end{array}$ & $\begin{array}{c}\text { Região sul } \\
\%\end{array}$ & P-Valor \\
\hline $\begin{array}{l}\text { Categoria } \\
\text { administrativa }\end{array}$ & & & & & 0,066 \\
\hline Públicas & 19 & 17 & 13,2 & 23 & \\
\hline Privadas & 125 & 57 & 86,8 & 77 & \\
\hline $\begin{array}{l}\text { Inserção e } \\
\text { oferta }\end{array}$ & & & & & 0,668 \\
\hline Presente & 88 & 43 & 61,1 & 58,1 & \\
\hline Ausente & 56 & 31 & 38,9 & 41,9 & \\
\hline $\begin{array}{l}\text { Natureza da } \\
\text { disciplina }\end{array}$ & & & & & 0,499 \\
\hline Obrigatória & 65 & 35 & 87,8 & 83,3 & \\
\hline Não obrigatória & 9 & 7 & 12,2 & 16,7 & \\
\hline $\begin{array}{l}\text { Metodologia de } \\
\text { ensino }\end{array}$ & & & & & 0,309 \\
\hline $\begin{array}{l}\text { Conteúdo } \\
\text { teórico }\end{array}$ & 35 & 7 & 64,8 & 50 & \\
\hline $\begin{array}{l}\text { Conteúdo } \\
\text { teórico-prático }\end{array}$ & 19 & 7 & 35,2 & 50 & \\
\hline
\end{tabular}

Legenda: N: número de Instituições de Ensino Superior (IES). Prevalência: percentual de IES de acordo com as regiões brasileiras. Teste qui-quadrado; significativo se $p<0,05$.

Fonte: Faria LV, et al., 2019.

Tabela 3 - Prevalência da disciplina de implantodontia de acordo com a natureza administrativa das IES de odontologia das regiões sul e sudeste do Brasil, 2019.

\begin{tabular}{|c|c|c|c|c|c|}
\hline \multirow[b]{2}{*}{ VARIÁVEIS } & \multicolumn{2}{|c|}{$\mathbf{N}$} & \multicolumn{2}{|c|}{ Prevalência (\%) } & \multirow[b]{2}{*}{ P-Valor } \\
\hline & $\begin{array}{c}\text { IES } \\
\text { pública }\end{array}$ & $\begin{array}{c}\text { IES } \\
\text { privada }\end{array}$ & IES pública & $\begin{array}{c}\text { IES } \\
\text { privada }\end{array}$ & \\
\hline Inserção e Oferta & & & & & $<0,001$ \\
\hline Presente & 31 & 100 & 86,1 & 54,9 & \\
\hline Ausente & 5 & 82 & 13,9 & 45,1 & \\
\hline Natureza da Disciplina & & & & & $<0,001$ \\
\hline Obrigatória & 21 & 79 & 67,7 & 92,9 & \\
\hline Não obrigatória & 10 & 6 & 32,3 & 7,1 & \\
\hline Metodologia de Ensino & & & & & 0,723 \\
\hline Conteúdo teórico & 13 & 29 & 65 & 60,4 & \\
\hline $\begin{array}{l}\text { Conteúdo teórico- } \\
\text { prático }\end{array}$ & 7 & 19 & 26,9 & 73,1 & \\
\hline
\end{tabular}

Legenda: N: número de Instituições de Ensino Superior (IES). Prevalência: percentual de IES de acordo com a categoria administrativa das IES. Teste qui-quadrado; significativo se $p<0,05$.

Fonte: Faria LV, et al., 2019. 
Se tratando da (V) carga horária média da disciplina de Implantodontia, 140 IES ofereceram essa informação. 70 IES ofereceram essa informação no sudeste brasileiro. Já no Sul, essa informação estava presente na matriz curricular de 40 faculdades. O estado que apresentou a maior $\mathrm{CH}$ média foi SP $(76,4 \mathrm{~h})$, seguido pelo ES (70,0h), RJ (57,5h), RS (62,0h), PR (61,1h), MG (52,4h) e SC (46,2h) (Gráfico 1.A). Do ponto de vista regional, as IES do sudeste apresentam uma $\mathrm{CH}$ maior, quando comparadas as da região sul (62,8 e 57,7h respectivamente) (Gráfico 1.B).

Gráfico 1 - Carga horária média da Implantodontia dos cursos de graduação das IES, por estados da federação $(A)$ e região do país $(B)$.

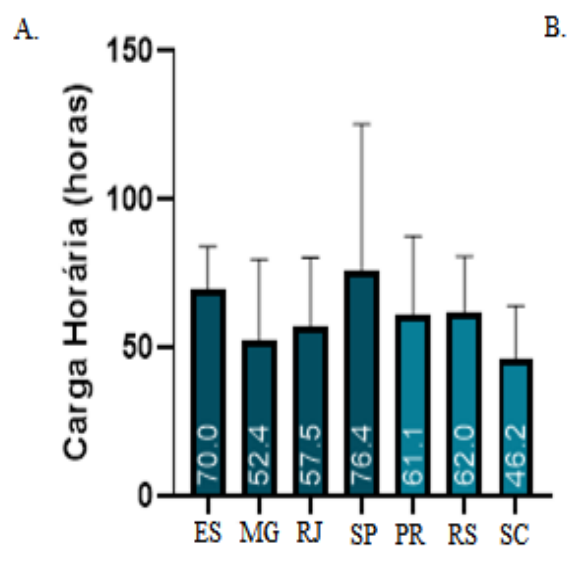

B.

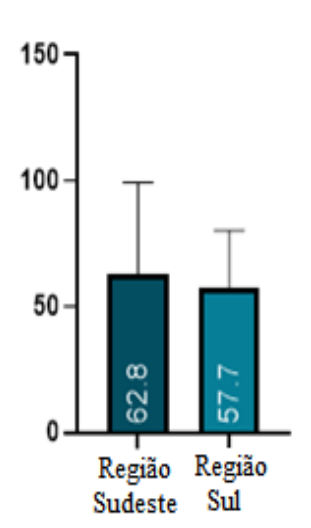

Fonte: Faria LV, et al., 2019.

A média foi de $60,97 \mathrm{~h}$ e a mediana de $60 \mathrm{~h}$ conforme destacado no Gráfico 2. Quanto à distribuição por categoria administrativa a $\mathrm{CH}$, para as públicas é de $53,29 \mathrm{~h}(\mathrm{DP}=23,943)$ e para as privadas de $63,99 \mathrm{~h}$ ( $D P=34,627)$, mas essa diferença não é significativa $(p=0,287)$. Como apresentado no Gráfico 3 , a carga horária média das disciplinas é apresentada de acordo com o tipo de conteúdo. Para as disciplinas apenas teóricas, a CH média foi de 49,44h (DP=22,019), enquanto para as disciplinas teórico-práticas, foi de 76,23h ( $D P=24,07)$, sendo $p=0,560$.

Gráfico 2 - Carga horária da implantodontia dos cursos de graduação das IES, por categoria administrativa das IES.

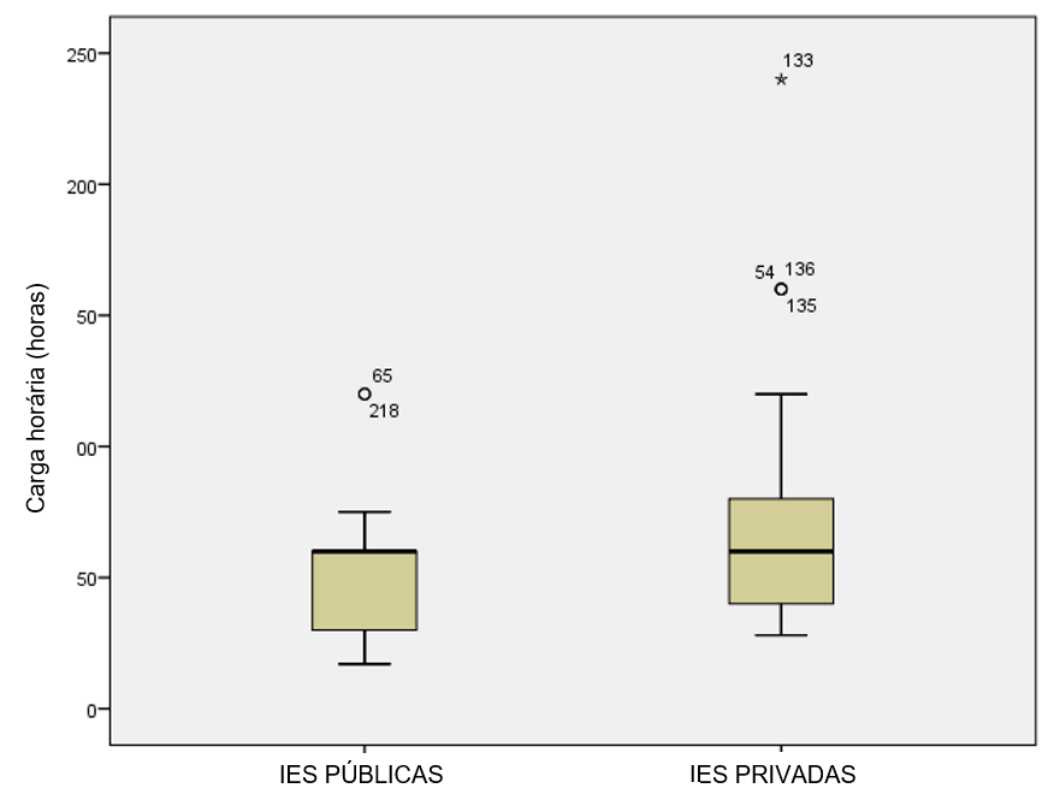

Fonte: Faria LV, et al., 2019. 
Gráfico 3 - Carga horária da implantodontia dos cursos de graduação das IES, por tipo de conteúdo ministrado.

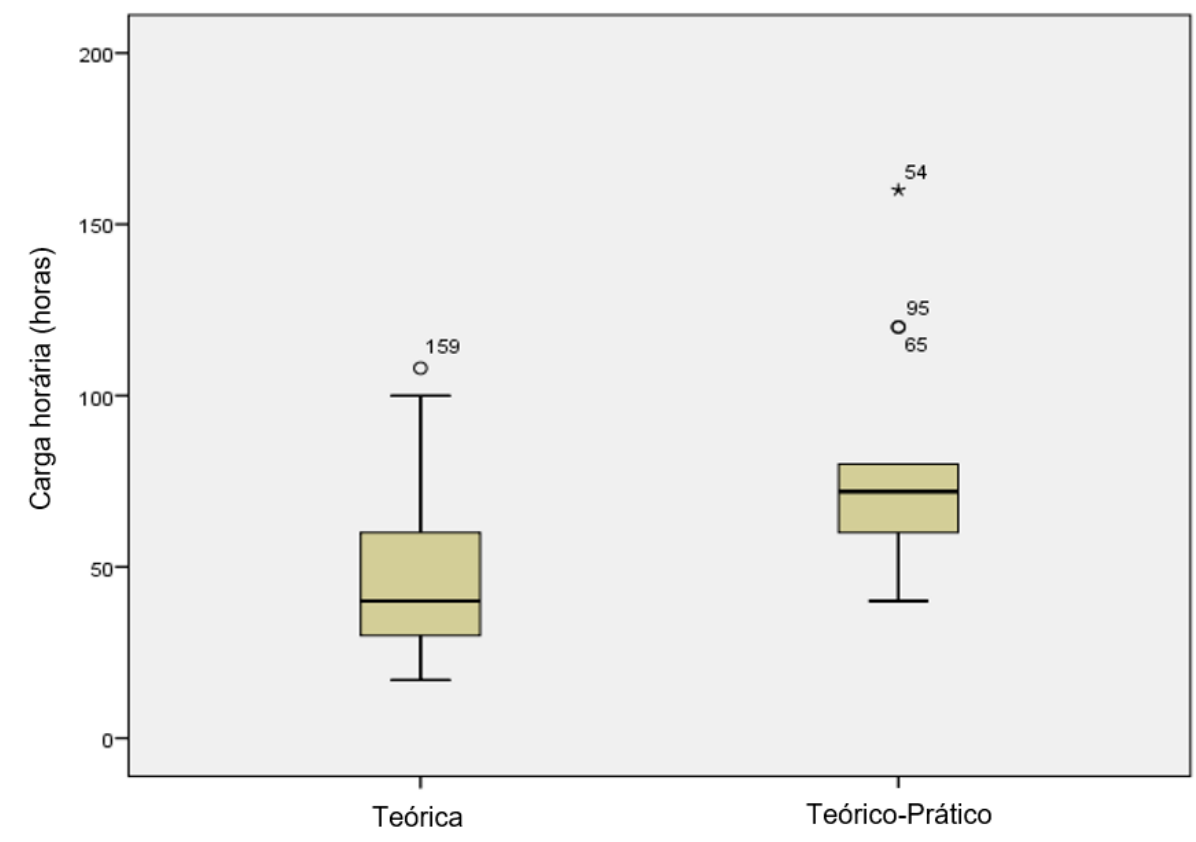

Fonte: Faria LV, et al., 2019.

\section{DISCUSSÃO}

Embora no Brasil os cursos de pós-graduação estejam cada vez mais disponíveis, levando a implantodontia a ser a terceira especialidade odontológica com maior número de profissionais registrados no Conselho Federal de Odontologia (CFO, 2019), é notória a necessidade de incorporar na formação de graduação conceitos dessa especialidade.

Barwacz CA, et al. (2015) destacam que embora seja realizada há mais de quatro décadas, a incorporação da implantodontia na graduação odontológica tem sido marcadamente conservadora quando comparada com outras modalidades de tratamento e avanços tecnológicos. De fato, a terapia reabilitadora com implantes pode exigir um conjunto de habilidades novas e particulares de um clínico. Especificamente, no nível prático, exigiria competências em habilidades cirúrgicas (LANG NP, et al., 2011). Em resposta a isso, foram realizadas reuniões em todo o mundo para estabelecer algum consenso no campo quanto aos caminhos educacionais e competências de referência associadas a implantodontia em nível de graduação.

Instituições odontológicas acadêmicas europeias (LANG NP e DE BRUYN H, 2009) e australianas (MATTHEOS N e IVANOVSKI S, 2010; MATTHEOS N, et al., 2010) reconheceram a necessidade de maior inclusão da terapia por implantes na graduação, onde a educação em implantes dentários e terapias de reabilitação como próteses sobre implantes são possíveis e com altas taxas de sucesso (KROEPLIN BS e STRUB JR, 2011).

Diferentemente, um fórum do sudeste asiático que contou com a presença de representantes de sete países, estabeleceu metas mais realistas para o ensino universitário da região, discordando dos consensos observados nos workshops europeus e australianos, ao concluírem que a colocação cirúrgica de implantes em pacientes estava além do nível de educação odontológica na graduação e era mais apropriada para os níveis de pós-graduação. Do mesmo modo, foram mais pragmáticos ao questionar os níveis de habilidades propostos pelos demais workshops no que diz respeito às competências dos clínicos acerca da reconstrução protética de implantes e da manutenção de pacientes com implantes, destacando que uma expectativa mais prática e razoável estaria no "nível de conhecimento sobre o assunto" e não em uma etapa de "deve ser competente em" (LANG NP, et al., 2010). 
Embora esses workshops não reflitam a realidade da educação de países em desenvolvimento com o Brasil, é indiscutível a necessidade da incorporação do conteúdo de implantodontia nas faculdades de odontologia brasileiras, na qual uma abordagem mais objetiva como destacada no fórum do sudeste asiático talvez seja mais condizente com a realidade do país.

Outras pesquisas curriculares que avaliaram a inserção da disciplina de implantodontia internacionalmente, observaram situações bem diferentes da relatada na pesquisa de 2010 realizada no Brasil (RABELO CC, et al., 2010). Nos Estados Unidos, foi observado um aumento na oferta, passando de $65 \%$ no início da década de 90 (ARBREE NS e CHAPMAN RJ, 1991), para 84\% em 2002 (LIM MV, et al., 2005). Outro estudo publicado em 2006 indicou que $97 \%$ das IES de odontologia nos EUA e no Canadá ofereciam experiência didática relacionada a implantes dentários e $86 \%$ forneciam alguma experiência clínica em implantes para seus alunos (PETROPOULOS VC, et al., 2006).

Um estudo que avaliou características dos programas de implantes dentários nas graduações canadenses observou que a maioria das instituições $(90 \%)$ integra formalmente seu programa de implantes no terceiro ano do currículo (BARWACZ CA, et al., 2016). No Reino Unido e Irlanda também foram observados um aumento na oferta, passando de $87 \%$ em um estudo realizado em 2007 (ADDY LD, et al., 2008), para 100\% dez anos depois em estudo realizado em 2017, na qual todas as escolas de odontologia incluídas oferecem treinamento em implantes para seus graduandos (CHIN JS, et al., 2018).

Uma pesquisa que avaliou escolas europeias de odontologia, concluída em 2002 e publicada em 2005, indicou que $75 \%$ das escolas respondentes haviam estabelecido programas de odontologia para implantes, mas apenas $37 \%$ relataram que seus alunos de graduação adquiriram experiência clínica com restaurações sobre implantes (AFSHARZAND Z, et al., 2005).

Quando comparadas as matrizes curriculares de IES da Europa e da América do norte a partir de estudos publicados nos anos 2000 (LIM MV, et al., 2005; AFSHARZAND Z, et al., 2005; PETROPOULOS VC, et al., 2006; ADDY LD, et al., 2008), observa-se que as IES do sul e sudeste do país possuem currículos defasados mesmo em 2019, uma vez que, apenas $58 \%$ das instituições ofertam a disciplina em seus programas de ensino. O maior problema parece estar nas instituições brasileiras privadas, uma vez que a prevalência da disciplina de implantodontia nas IES públicas é cerca de $86 \%$, semelhante ao padrão europeu.

Uma revisão sistemática realizada em 2014 explorou a implementação dos programas educacionais de implantodontia nos currículos de graduação e observou que, em geral, três componentes didáticos na educação sobre implantes podem ser identificados. O ensino teórico, é o mais comum, e é oferecido para fornecer aos alunos o conhecimento dos princípios básicos da odontologia de implantes ou faz parte da linha de aprendizado para fundamentar a educação pré-clínica e clínica. O segundo componente, os exercícios pré-clínicos, têm uma frequência menor, e o terceiro, a educação clínica no contexto de assistência ou auto tratamento relacionado ao implante, é oferecida apenas em uma quantidade limitada de programas (KOOLE S e DE BRUYN, 2014).

Uma revisão de literatura realizada em 2011 (KROEPLIN BS e STRUB JR, 2011), avaliou a integração da odontologia de implantes nos currículos de graduação em todo o mundo nas últimas décadas, ao incluírem publicações de 1974 a 2009. Os autores observaram que a implantodontia como parte dos currículos de graduação, é predominantemente ministrada por cursos teóricos na forma de palestras, com duração de 1 a 20 horas. Outro importante achado é que a terapia de implante integrada no treinamento pré-clínico e clínico foi menos aparente e diferiu bastante entre os currículos.

Nossos resultados também demostram uma grande heterogeneidade nas cargas horárias das disciplinas teóricas e teórico-práticas relacionadas a implantodontia. Embora seja uma limitação do nosso estudo não poder distinguir como é abordada a metodologia de ensino prática relatada nas matrizes curriculares, observase que menos da metade das instituições buscam incorporar aos seus programas alguma atividade prática. Obviamente, nossos resultados apontam que quando a disciplina é teórico-prática a $\mathrm{CH}$ é maior, com cerca de 5 créditos $(75 \mathrm{~h})$ em média, já para as disciplinas apenas teóricas a $\mathrm{CH}$ é menor, cerca de 3 a 4 créditos (próximo a 50h). Realidade muito diferente se faz nos currículos norte-americanos, onde um estudo 
demostrou que, além do componente didático, programas de implantes a nível de graduação oferecem treinamento clínico aos estudantes por meio de exercícios pré-clínicos simulados em $90,4 \%$ e assistência direta supervisionada ao paciente em 94,2 (CHRISTOPHER A, 2015). No Canadá, de forma semelhante, os currículos incluem exercícios simulados e atendimento direto ao paciente sob supervisão em $90 \%$ dos programas (BARWACZ CA, et al., 2016). No Reino Unido e Irlanda, a maioria das escolas oferece aos alunos experiência clínica direta no planejamento do tratamento, no entanto, a experiência clínica direta em restaurações sobre implantes e na colocação do implante ainda é baixa (CHIN JS, et al., 2018).

Christopher A, et al. (2015) destacam que programas que oferecem essas experiências de aprendizagem resultam em níveis de confiança mais altos e maior probabilidade de incorporar a terapia de implantes em sua prática após a graduação, como também ressaltado por outros trabalhos (YUAN JC, et al., 2011), o que torna a inclusão generalizada da experiência clínica, pré-clínica e supervisionada uma tendência nos currículos de graduação. Por outro lado, Afsharzand Z et al. (2005) destacam que o objetivo do ensino de graduação é fornecer aos pacientes conselhos inteligentes sobre indicação e usos de implante, ao invés de fornecer aos alunos treinamento suficiente para permitir a execução do tratamento. No Brasil, ainda é discreta a inserção na disciplina Implantodontia nos currículos de graduação, passando de uma situação em 2008, na qual metade das escolas brasileiras ainda não possuíam nos currículos a disciplina de implantodontia (RABELO CC, et al., 2010) para apenas 60,09\% em 2019 nas regiões sul e sudeste do Brasil.

Os resultados deste estudo reforçam a necessidade de remodelar os currículos no que diz respeito ao ensino de implantodontia nas graduações de ensino superior de odontologia no Brasil, podendo auxiliar os coordenadores dos cursos em futuras reformulações curriculares, dada a importância do assunto. Um fator facilitador seria a realização de um encontro entre instituições representativas brasileiras, como a Associação Brasileira de Ensino Odontológico (ABENO) e o Conselho Federal de Odontologia, na qual seja desenvolvido um documento de consenso sobre o ensino da implantodontia no currículo de graduação (e pós-graduação) das escolas odontológicas brasileiras, assim como já feito em outros países (LANG NP e DE BRUYN H, 2009; MATTHEOS N e IVANOVSKI S, 2010; MATTHEOS N, et al., 2010), a fim de nortear reorganizações curriculares futuras e de homogeneizar o ensino de implantodontia ao nível de graduação.

\section{CONCLUSÃO}

A partir da análise das matrizes curriculares das IES do sul e sudeste do Brasil observou-se que IES privadas ofertam menos a disciplina de implantodontia que IES públicas, e que, quando ofertada, a tendência da disciplina é ser predominantemente obrigatória, em ambas. Além disso, observou-se que poucas instituições, especialmente as públicas, inserem atividades práticas de implantodontia em seus programas de graduação. É necessário uma reformulação e padronização dos currículos odontológicos no que tange o ensino de implantodontia das graduações de odontologia. De face a grande aceitação clínica e o aumento da demanda de pacientes por implantes dentários os programas educacionais das escolas de odontologia devem dar subsídios aos alunos para conhecerem e indicarem esta modalidade de reabilitação.

\section{REFERÊNCIAS}

1. ADDY LD, et al. The teaching of implant dentistry in undergraduate dental schools in the United Kingdom and Ireland. Br Dent J. 2008 Dec 13;205(11):609-14.

2. AFSHARZAND Z, et al. Predoctoral implant dentistry curriculum survey: European dental schools. Eur J Dent Educ 2005; 9:37-45.

3. ARBREE NS, CHAPMAN RJ. Implant education programs in North American dental schools. J Dent Educ. 1991 Jun;55(6):378-80.

4. BARWACZ CA, et al. An overview of U.S. predoctoral dental implant programs and their directors. J Dent Educ. 2015 Mar;79(3):265-77.

5. BARWACZ CA, et al. Comparison of Canadian and united states predoctoral dental implant education. J Can Dent Assoc. 2016 Aug;82:g22.

6. BRÄGGER U, et al. Economic aspects of single-tooth replacement. Clin Oral Implants Res. 2005 Jun;16(3):335-41.

REAS/EJCH | Vol.12(4) | e2672 | DOI: https://doi.org/10.25248/reas.e2672.2020 Página 9 de 10 
7. BRASIL. Ministério da Educação. Cadastro e-MEC de Instituições e Cursos de Educação Superior. Disponível em: http://emec.mec.gov.br/. Acesso em 8 março de 2019, 2019.

8. BRASIL. Ministério da Educação. Portaria Normativa ํㅡ 21, de 21 de dezembro de 2017. Dispõe sobre o sistema eMEC, sistema eletrônico de fluxo de trabalho e gerenciamento de informações relativas aos processos de regulação, avaliação e supervisão da educação superior no sistema federal de educação, e o Cadastro Nacional de Cursos e Instituições de Educação Superior Cadastro e-MEC. Diário Oficial da União. 22 de dez 2017; Seção 1:18-19-31.

9. CHIN JS, et al. Teaching of implant dentistry in undergraduate dental schools in the UK and Ireland. Br Dent J. 2018 Oct ; 225:763-768.

10. CHRISTOPHER A, et al. An Overview of U.S. Predoctoral Dental Implant Programs and Their Directors. Journal of Dental Education March 2015, 79 (3) 265-277.

11. CONSELHO FEDERAL DE ODONTOLOGIA (CFO). Quantidade Geral de Cirurgiões-Dentistas Especialistas.

12. DRAGAN IF, et al. A global perspective on implant education: Cluster analysis of the "first dental implant experience" of dentists from 84 nationalities. Eur J Dent Educ. 2019 Aug;23(3):251-265.

13. FILLION M, et al. The impact of implant treatment on oral health related quality of life in a private dental practice: a prospective cohort study. Health Qual Life Outcomes 2013;11:197.

14. JAHANGIRI L. Implant dentistry in predoctoral education: where are we? J Dent Educ. 2015 Mar;79(3):239-40.

15. KOOLE S, DE BRUYN H. Contemporary undergraduate implant dentistry education: a systematic review. Eur J Dent Educ. 2014 Mar;18 Suppl 1:11-23.

16. KROEPLIN BS, STRUB JR. Implant dentistry curriculum in undergraduate education: part 2-program at the AlbertLudwigs University, Freiburg, Germany. Int J Prosthodont. 2011 Nov-Dec;24(6):544-56.

17. KROEPLIN BS, STRUB JR. Implant dentistry curriculum in undergraduate education: part 1-a literature review. Int J Prosthodont. 2011 May-Jun;24(3):221-34.

18. LANG NP, DE BRUYN H; 1st European Consensus Workshop in Implant Dentistry University Education. The rationale for the introduction of implant dentistry into the dental curriculum. Eur J Dent Educ. 2009 Feb;13 Suppl 1:19-23.

19. LANG NP, et al. Implant dentistry in undergraduate dental curricula in South-East Asia: forum workshop at the University of Hong Kong, Prince Philip Dental Hospital, 19-20 November 2010. J Investig Clin Dent. 2011 Aug;2(3):1525.

20. LIM MV, et al. Predoctoral implant education in U.S. dental schools. J Prosthodont. 2005 Mar;14(1):46-56.

21. MARTIN ASS, et al. Distribuição dos cursos de Odontologia e de cirurgiões-dentistas no Brasil: uma visão do mercado de trabalho. Revista da ABENO. 2018;18(1):63-73.

22. MATTHEOS N, et al. University teaching of implant dentistry: guidelines for education of dental undergraduate students and general dental practitioners. An Australian consensus document. Aust Dent J. 2010 Sep;55(3):329-32.

23. MATTHEOS N, IVANOVSKI S. Implant Dentistry and the University: an Australian model. Australian Consensus Workshop on Implant Dentistry University Education, Gold Coast, 4-6 February 2010. Introduction. Aust Dent J. 2010 Sep;55(3):328

24. PAPASPYRIDAKOS $P$, et al. Implant and prosthodontic survival rates with implant fixed complete dental prostheses in the edentulous mandible after at least 5 years: a systematic review. Clin Implant Dent Relat Res 2014;16(5):705-17.

25. PETROPOULOS VC, et al. Teaching implant dentistry in the predoctoral curriculum: a report from the ADEA implant workshop's survey of deans. J Dent Educ 2006;70(5):580-8.

26. PLASSCHAERT AJ. Association for Dental Education in Europe. Profile and competences for the European dentist. Eur J Dent Educ. 2005 Aug;9(3):98-107.

27. RABELO CC, et al. Análise transversal do ensino da implantodontia no curso de graduação. Revista da ABENO. 2010;10(2):53-58.

28. SANZ M, SAPHIRA L; 1st European Consensus Workshop in Implant Dentistry University Education. Competencies in implant therapy for the dental graduate: appropriate educational methods. Eur J Dent Educ. 2009 Feb;13 Suppl 1:3743.

29. VANDEWEGHE S, et al. Dental implants placed by undergraduate students: clinical outcomes and patients'/students' perceptions. Eur J Dent Educ. 2014 Mar;18 Suppl 1:60-9.

30. YUAN JC, et al. Dental student perceptions of predoctoral implant education and plans for providing implant treatment. J Dent Educ 2011;75(6):750-60. 